\title{
Arno Engelmann (1931-2017) Ein Gestaltpsychologe in Brasilien
}

Arno Engelmann wurde am 6.12.1931 in Berlin geboren. Der Machtantritt des Nationalsozialismus beraubte seine Familie wie so viele andere deutsche Juden ihrer Heimat. Die Flucht führte sie zuerst nach Frankreich, 1941 dann weiter nach Brasilien.

Arno Engelmann wäre gerne Pianist geworden, doch sein Vater bestand auf einer traditionellen Ausbildung. So begann er 1952 ein Medizinstudium an der Universität von São Paulo (USP). 1955 wechselte er zur Philosophie, in deren Studium damals auch noch die Psychologie integriert war. Dort kam er mit der Gestaltpsychologie in Kontakt, für die er rasch Feuer fing. Dafür war vor allem der Einfluss seiner Lehrerin Annita Cabral maßgeblich:

Annita Cabral (Annita de Castilho e Marcondes Cabral, 1911-1991) war von 1947 bis 1968 Professorin und Inhaberin des Lehrstuhls für Psychologie im Philosophie-Studium der USP. Sie betrachtete sich Zeit ihres Lebens als Gestaltpsychologin, wie Engelmann in seiner Einführung zur „Geschichte der Psychologie in Brasilien" (2004) hervorhebt. Cabral hatte 1941 ursprünglich am Smith College bei Kurt Koffka studiert. Nachdem aber Koffka noch im selben Jahr gestorben war, wechselte sie für die beiden Folgejahre an die Graduate Faculty der New School for Social Research, wo sie bei Max Wertheimer ihre Master-Arbeit zum Thema "Memória das Formas" / "Memory of Forms" ausarbeitete. Engelmann fand in Cabral eine überzeugte und überzeugende Mentorin für seine Beheimatung in der Ideenwelt und Methodik der Gestaltheorie.

1960 wurde Engelmann eine Universitäts-Assistentenstelle für Philosophie angeboten, aber auch eine für Psychologie. Er entschied sich für die Psychologie, um weiter der Gestaltpsychologie nachgehen und experimentell arbeiten zu können. 1961 verbrachte er ein Forschungssemester in den USA an der Universität von Illinois. Nach seiner Rückkehr 1962 wurde er dem Psychologie-Lehrstuhl der USP zugeteilt, der später zur Abteilung für Experimentelle Psychologie wurde. 1972 machte Engelmann sein Doktorat, 1991 habilitierte er sich, 1993 wurde er Titular-Professor für Psychologie an der USP (in Brasilien entspricht das dem full professor bzw. dem Ordinarius).

1978 war insofern ein wesentliches Jahr in Engelmanns wissenschaftlicher Tätigkeit, als in diesem Jahr zwei seiner wichtigsten Buchpublikationen erschienen. Dies war zum einen eine Wolfgang Köhler-Anthologie, zum anderen 
seine Forschungsarbeit „Os Estados Subjetivos. Uma Tentativa de Classificação de seus Relatos Verbais".

Vor Engelmanns Köhler-Anthologie war in Brasilien das Werk Wolfgang Köhlers in portugiesischer Sprache nur durch eine 1968 erschienene Übersetzung seiner "Gestalt Psychology“ aus 1929 zugänglich gewesen (deutsche Fassung 1933: „Psychologische Probleme“). Engelmanns Anthologie machte nun erstmals einen umfassenderen Einblick in die grundlegenden Beiträge Köhlers zur Gestaltpsychologie möglich. Das Buch enthält eine Einführung von Engelmann, in der er - nach einer Skizze des Lebenslaufs von Köhler - dessen Gesamtwerk in seinen inhaltlichen Grundzügen vorstellt. Dem folgt eine ausführliche Bibliographie. Der Auswahlteil ist in drei Abschnitte gegliedert, die Engelmann die erste, zweite und dritte Periode des Schaffens Köhlers bezeichnet. Zur ersten Periode (1909-1934) bringt er Übersetzungen von Ausschnitten der „Intelligenzprüfungen an Anthropoiden“ und „Gestaltprobleme und Anfänge einer Gestalttheorie“; für die zweite Periode (1935-1945) einen Ausschnitt über die Isomorphie-These aus „Werte und Tatsachen“ sowie den Aufsatz „Value and Fact“ (1944); aus der dritten Periode (1946-1967) „The Mind-Body Problem“ (1960), „Gestalt Psychology Today“ (1961), „Recent Developments in Gestalt Psychology“ (ein Kapitel aus „The Task of Gestalt Psychology“ 1969) sowie „Perception and Attention“ (1958).

Engelmanns zweites Buch aus dem Jahr 1978, „Os Estados Subjetivos. Uma Tentativa de Classificação de seus Relatos Verbais”, präsentiert seine Forschungsarbeiten zu einem Themengebiet, das auch für sein weiteres Forscherleben zentral bleiben sollte: Den Buchtitel könnte man übersetzen mit „Die subjektiven Zustände. Versuch einer Klassifikation ihrer Verbalisierungen“. Es berichtet über eine breit angelegte Untersuchung der alltagssprachlichen Verbalisierung von Gefühlszuständen: Sie stützt sich auf die Klassifikation von 370 verbalen Ausdrücken für unterschiedliche Gefühlszustände durch 2249 Personen in São Paulo. Die Betonung des Titels der Studie liegt zum einen auf „subjektiv “, da es sich bei Gefühlszuständen immer nur um dem individuellen Subjekt unmittelbar zugängliche Zustände handelt; zum anderen auf „Zustände“ des Subjekts, da Engelmann mit seiner Untersuchung nicht linguistische Zielsetzungen verfolgt, sondern psychologische: die erfasste Vielfalt an Verbalisierungen soll einen Weg zur Erfassung und Ordnung der Vielfalt an subjektiven Gefühlszuständen bahnen.

2005 nahm Engelmann erstmals persönlich an einer internationalen Tagung der GTA teil, nämlich an der 14. Wissenschaftlichen Arbeitstagung an der Universität Graz mit dem Rahmenthema „Werte, Sinn und Tatsachen. Der Mensch als Subjekt" (Boudewijnse 2006). In seinem Vortrag „The percept echelons consciousness proposal“ spannte er den Bogen von den eben angeführten früheren Forschungen zur Erfassung subjektiver Gefühlszustände bis zu seinem über die 
Jahrzehnte weiter ausgearbeiteten Konzept des „unmittelbaren und mittelbaren Bewusstseins" (immediate consciousness / mediate consciousness):

Der „Primat des Phänomenalen“, wie er in erkenntnistheoretischer Hinsicht in der Gestalttheorie vertreten wird, fordert nach Engelmann ein entsprechendes Verständnis des „unmittelbar phänomenal Gegebenen“. Dieses ist erstens immer nur der jeweiligen Person gegeben, es ist also individuell, und zweitens ist es immer nur das gegenwärtig Gegebene, ist demnach von nur sehr kurzer zeitlicher Dauer (im Allgemeinen etwa 3 Sekunden). Von diesem unmittelbaren Bewusstsein ist das mittelbare zu unterscheiden: sei es das unmittelbar phänomenal Gegebene anderer Personen, sei es auch das eigene, soweit es außerhalb dieser 3-Sekunden-Spanne des Gegenwärtigen, also bereits in der Vergangenheit liegt oder sich auf Zukünftiges bezieht. Die verschiedenen Zustände, die das Wahrnehmungsbewusstsein annehmen kann, sind nach dem Vorschlag Engelmanns als eine Staffelung von schwächeren und stärkeren Gestalten zu verstehen, die das Bewusstsein in der einen und anderen Richtung durchläuft (Engelmann 2006).

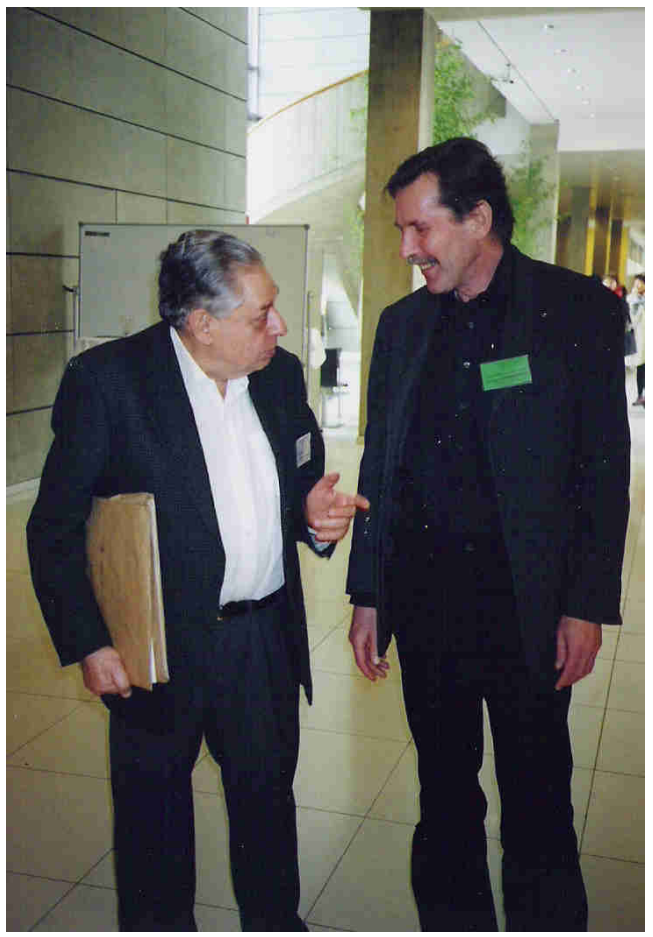

Das Foto zeigt Arno Engelmann 2005 bei der GTA-Tagung an der Universität Graz, im Gespräch mit G. Stemberger, damals Vorsitzender der GTA
Gemeinsam mit Nilson Guimarães Doria verfasste Engelmann 2007 für die Zeitschrift Gestalt Theory einen Übersichtsbeitrag zu Geschichte und Gegenwart der Gestalttheorie in Brasilien. Im gleichen Jahr nahm er mit seiner Frau, der Psychoanalytikerin Lucy Engelmann, ein weiteres Mal an einer internationalen wissenschaftlichen Arbeitstagung der GTA teil, „Relations and Structures - Developments of Gestalt Theory in Psychology and Adjacent Fields" an der Universität Macerata, bei der er „Starting from the immediateness" vortrug. Die von ihm vorgetragenen Überlegungen schlugen sich im Jahr darauf in einem Beitrag und einer damit verbundenen Kontroverse in der Zeitschrift Integrative Psychological and Behavioral Science nieder: 
Das Verständnis des unmittelbar gegebenen Bewusstseins als zugleich immer nur individuell und immer nur flüchtig führte Engelmann zu der Frage, wie der Mensch die damit verbundene Einsamkeit ertragen und mit anderen Menschen überhaupt etwas Gemeinsames finden kann. Dies wurde nach seinem Vortrag in Macerata Gegenstand eines Artikels mit dem bezeichnenden Titel (Übers. GSt): „Von der entsetzlichen Einsamkeit zur wunderbaren Übereinstimmung von Menschen“ („From the terrible loneliness to the wonderful agreement of human beings", Engelmann 2008a). Seine Antwort auf diese Frage, die er dann in einer Kontroverse mit dem Entwicklungspsychologen und Psychoanalytiker Roger Bibace von der Clark University verteidigte (Bibace 2008, Engelmann 2008b), gründete er auf seinen „probabilistischen Skeptizismus“: Wäre dem Menschen die Tatsache bewusst, dass das ihm unmittelbar gegebene Bewusstsein immer nur individuell und immer nur flüchtig ist, müsste ihn eine grauenhafte Einsamkeit überkommen. Aber im gegebenen Moment ist sich der Mensch dieser Tatsache praktisch nie gewahr - im Gegenteil: Er erlebt sich in einer Wirklichkeit umgeben von vielerlei Menschen und beteiligt an vielerlei Ereignissen. Würde er das nun kritisch hinterfragen, käme er zum Ergebnis, dass diesen Ereignissen außerhalb seines eigenen unmittelbaren Bewusstseins zwar mit einer gewissen Wahrscheinlichkeit etwas entspricht (unter anderem auch im Bewusstsein anderer Menschen), aber eben nur mit einer gewissen Wahrscheinlichkeit. Er hat es also, kritisch betrachtet, mit Hypothesen zu tun, nicht mit absoluten Realitäten. Da dies bei allen Menschen so ist, besteht jedoch die Möglichkeit der Übereinstimmung und Gemeinschaft. Engelmann sah diese seine Position des probabilistischen Skeptizismus als mit der Gestalttheorie vereinbar an, auch wenn ihm bewusst war, dass viele Gestalttheoretiker diese Auffassung nicht teilen würden: "Engelmann is a disciple of Gestalt theory which is radically different from Descartes' type of theory. But on the other hand, Engelmann is a probabilistic skeptic." (Engelmann 2008b, S. 89)

Arno Engelmann starb am 3. November 2017 in seinem 85. Lebensjahr in São Paulo. Die Gestaltpsychologie und die ganze gestalttheoretische Gemeinschaft verlieren mit Arno Engelmann einen unverwechselbaren eigenständigen Denker und eine wichtige Stimme. Die GTA hat viele Gründe, um ihn zu trauern. Unser tiefes Mitgefühl gehört vor allem seiner Frau Lucy, die über viele Jahrzehnte ihr Leben mit ihm geteilt hat, seiner Familie und seinen Freunden.

\section{Gerhard Stemberger, Wien/Berlin}




\section{Literatur}

Bibace, Roger (2008). Comparative Evaluations. Integrative Psychological and Behavioral Science, 42(1), 76-86. Boudewijnse, Geert-Jan (ed., 2006). Das Mentale Paradoxon - The Mental Paradox. Wien: Krammer.

Engelmann, Arno (1978). Os estados subjetivos: uma tentativa de classificaçâo de seus relatos verbais. São Paulo: Editora Ática.

Engelmann, Arno (ed., 1978). Wolfgang Köhler - Psicologia. São Paulo: Editora Ática.

Engelmann, Arno (2006). The Percept Echelons Consciousness Proposal. In: Boudewijnse 2006, 223-233.

Engelmann, Arno (2008a). From the terrible loneliness to the wonderful agreement of human beings. Integrative Psychological and Behavioral Science, 42(1), 56-75.

Engelmann, Arno (2008b). Two Important but Almost Never Related Beliefs. Integrative Psychological and Behavioral Science, 42(1), 87-91.

Engelmann, Arno \& Nilson Guimarães Doria (2007). Gestalt Theory in Brazil. Gestalt Theory, 29(3), 248-255. Volltext im Internet: http://www.gestalttheory.net/cms/uploads/pdf/archive/2007Engelmann_Doria_GTHinBrazil.pdf

Engelmann, Arno \& Renato Rodrigues Kinouchi (2009). Entrevista com Arno Engelmann. Scientiae Studia, 7(2). WWW: http://www.scielo.br/scielo.php?script=sci_arttext\&pid=S1678-31662009000200011

Gerhard Stemberger, Dr.phil., geb. 1947 in Innsbruck, Soziologe und Psychotherapeut, Lehrtherapeut für Gestalttheoretische Psychotherapie (GTP). Forscht und publiziert vorwiegend über die Grundlagen und klinischen Anwendungen der Gestalttheorie in der Psychotherapie. Mit-Herausgeber der Zeitschrift "Phänomenal - Zeitschrift für Gestalttheoretische Psychotherapie", langjähriger geschäftsführender Herausgeber und nunmehr Mitglied des Honorary Board der Gestalt Theory.

Adresse: A-3002 Purkersdorf, Wintergasse 75-77/7, Österreich.

E-mail: gst@gestaltheory.net 